\title{
Infection control awareness amongst illiterate workers in an Indian Intensive Care Unit: Impact of an unconventional educational intervention
}

\author{
Parikshit Singh ${ }^{1}$, Baronia AK ${ }^{1}$, Prasad KN² , Dhole TN² ${ }^{2}$ Singh SK ${ }^{2}$ \\ 1. Department of Critical Care Medicine, Sanjay Gandhi Post Graduate \\ Institute of Medical Sciences, Lucknow, Uttar Pradesh, India \\ 2. Department of Microbiology, Sanjay Gandhi Post Graduate \\ Institute of Medical Sciences, Lucknow, Uttar Pradesh, India
}

\author{
Key words \\ HEALTH CARE PERSONNEL - education; INFECTION CONTROL; INTENSIVE CARE UNITS; CROSS \\ INFECTION - prevention and control
}

\section{Introduction \\ The incidence of Hospital Acquired Infections (HAI) is directly linked with Infection control awareness. Prevention of HAI reduces morbidity, mortality, length of stay and cost of therapy among patients admitted in intensive care units (ICU). ${ }^{1}$ Illiteracy amongst patient helpers and cleaners is a well known barrier to implementing infection control by conventional educational methods in Indian hospitals.}

We aimed to improve infection control awareness amongst illiterate ICU workers by an unconventional educational intervention giving them a direct visual illustration of the potentially pathogenic microorganisms living on their hands and in their nares. The program used images from the New England Journal of Medicine. ${ }^{2}$ The impact of this intervention was assessed by verbal questionnaires on the worker's awareness of bugs and cross infection in the ICU before and after the intervention.

\section{Corresponding author}

Parikshit Singh MD

Department of Critical Care Medicine, Sanjay Gandhi Post Graduate Institute of Medical Sciences,

Lucknow, Uttar Pradesh, India

Email: drparikshitsingh@yahoo.co.in 


\section{Methods}

This study was carried out on illiterate workers in an ICU of a tertiary care university hospital in India. The workers, 15 patient helpers and cleaners, had never received any formal schooling. A questionnaire was designed in the native language containing 25 questions on basic awareness of infection, microbes, tools for infection control, hand hygiene and behavioural aspects - their ability to think rationally etc. Question-answer sessions were conducted verbally on an individual basis before and after a guided and programmed visit to the microbiology laboratory. The answers were noted by one or the other authors. Three authors evaluated and graded the answers as correct, partially correct or incorrect. Two points were given to a correct answer while 1 point and 0 points were awarded for partially correct and incorrect answers.

The questions asked in the questionnaire were:

1. At home how often do you wash your hands with plain soap and water and why?

2. At home how often do you wash your hands with water only (without soap) and why?

3. In ICU how often do you wash your hands with plain soap and water and why?

4. In ICU how often do you wash your hands with water only (without soap) and why?

5. In ICU how often do you clean your hands by hand-rub and why?

6. What is the difference in the way doctors wash their hands with plain soap and water before central venous catheter insertion and the way you wash your hands?

7. How will you like to dry your hands (hand-dryer, single use paper towel, multi-use cloth towel, air dry) after soap and water hand-wash and why?

8. What is the best use of unsterile latex gloves and when and how do you dispose them?

9. What is the best use of sterile latex gloves and when and how do you dispose them?

10. What is the best use of unsterile polyethylene gloves when and how do you dispose them?

11. What is the best use of sterile polyethylene gloves when and how do you dispose them?

12. What is the best use of unsterile plastic aprons and when and how do you dispose them?

13. What is the best use of sterile plastic aprons and when and how do you dispose them?

14. In order to save time on a busy day which of the following cleaning work would be deferred by you -1. Cleaning of side rails of bed, 2. Cleaning of side walls of room.

15. In order to save time on a busy day which of the following cleaning work would be deferred by you -1. Cleaning of computer keyboard, 2. Cleaning of computer screen.

16. What is the best way to dispose-off the contents of patients suction jar and why?

17. What is the best way to empty the urine bag and why?

18. What is the best way to handle urinary tube and bag when shifting the patient from one bed to the other and why?

19. What do you understand by bacteria?

20. How are you, me, patient and nurse inter-related with regard to transmission of bacteria from one to the other and to the patient?

21. Do you think that you are a key person who can reduce the transmission of bacteria from one to the other and to the patient?

22. How would the rings, bangles and watches that you wear harm the patient?

23. What is the importance of colour coding of waste disposal bags?

24. What is the common point in sneezing, coughing or talking loudly near the patient bed?

25. What practical problems do you face in cleaning an ICU cubicle with a patient inside and how do you overcome such problems?

In the microbiology laboratory:

1. A brief talk was given by a microbiologist about the role of invisible microorganisms (present on our body surface) in the spread of cross infection in the ICU using illustrations and posters. This talk aimed to make workers aware about the genesis of infection.

2. A senior laboratory technician made impressions of all five fingertips from one hand of each individual on a culture plate. They were then asked to have a good hand-rub and a second imprint was taken.

3. The following day the growth from their own hands on the culture plates before and after the rub was shown to them. The microbiologist also showed them the organisms under the microscope to help them visualise the pathogens and generate a lasting impression. 
Table I. Comparing pre- and post-intervention period

ICU Worker $(\mathbf{n}=15)$

Total Score (out of 50)

\begin{tabular}{|c|c|c|c|c|}
\hline & Age $(Y r)$ & $\operatorname{Sex}(M / F)$ & Pre-intervention & Post-intervention \\
\hline 1. & 27 & M & $32(64.0)$ & $41(82.0)$ \\
\hline 2. & 22 & M & $38(76.0)$ & $46(92.0)$ \\
\hline 3. & 47 & M & $43(86.0)$ & $49(98.0)$ \\
\hline 4. & 51 & M & $41(82.0)$ & $49(98.0)$ \\
\hline 5. & 25 & M & $37(74.0)$ & $42(84.0)$ \\
\hline 6. & 35 & M & $36(72.0)$ & $40(80.0)$ \\
\hline 7. & 25 & M & $15(30.0)$ & $36(72.0)$ \\
\hline 8. & 24 & M & $41(82.0)$ & $46(92.0)$ \\
\hline 9. & 45 & $\mathrm{~F}$ & $35(70.0)$ & $47(94.0)$ \\
\hline 10. & 25 & M & $37(74.0)$ & $42(84.0)$ \\
\hline 11. & 26 & M & $33(66.0)$ & $42(84.0)$ \\
\hline 12. & 25 & M & $35(70.0)$ & $44(88.0)$ \\
\hline 13. & 22 & M & $44(88.0)$ & $48(96.0)$ \\
\hline 14. & 50 & $\mathrm{~F}$ & $35(70.0)$ & $44(88.0)$ \\
\hline 15. & 24 & M & $34(68.0)$ & $45(90.0)$ \\
\hline \multicolumn{3}{|c|}{ Mean \pm SD } & $35.73 \pm 6.76$ & $44.06 \pm 3.63$ \\
\hline
\end{tabular}

Values in parentheses indicate percentages

4. Finally a debriefing session was conducted by the microbiology department, which included a talk about the relevance of the previous demonstrations. The importance of hand washing and the methods of breaking the chain of infection transmission were discussed using illustrations and posters.

\section{Results}

The total points scored out of a maximum of 50 (25 questions) were compared for the pre- and postintervention periods (Table I), and a statistically significant improvement in the scores was found post intervention in both the paired t-test $(\mathrm{t} p=7.499$, $\mathrm{p}=0.001)$ and the non-parametric Wilcoxon test $(z=3.42, p=0.001)$.

\section{Discussion}

Infection control awareness is usually brought about by educational programs using four key teaching methods: verbal (one-way) lecture, written (reading), visual-verbal (illustrated lectures) and participatory (role play, case study, practice etc.). ${ }^{3}$ These programs need reading-writing skills and so would not yield good results in our set-up.

The practice and method of hiring illiterate workers on contract as cleaners and patient helpers in Indian hospitals is unlikely to change. The authors were convinced intuitively that the images published by Curtis JD and Brittany $\mathrm{CE}^{2}$ have substantial visual educational content. To overcome illiteracy, the concept of these images was used to develop an 
educational program with verbal and visual contents only. The educational program was supported by the microbiology department. The efficacy of the program was tested by a questionnaire-based feedback. The practice of seeking feedback from healthcare workers using questionnaires has been found useful in the past. $^{4}$

No studies on illiteracy amongst ICU workers in relation to HAI have been published. We thought of this educational activity as a simple idea to generate interest in the mundane work schedule of an illiterate ICU worker and also to see the impact of the directly perceivable visual and verbal contents of our educational program.

The visual-verbal educational intervention showed significant objective improvement in the workers understanding about HAI. Besides objective improvement, we sensed a subjective postintervention improvement in day to day activities carried out by these workers in the form of increased hand washing, frequent changing of gloves and other protective barriers, and also an increase in enthusiasm and commitment while performing daily ICU chores. Clearly the illiterate workers could think actively about the presence of 'bugs' and connect well with their possible role in the prevention of HAI.

Further studies involving longer times and workers in other hospital departments are desirable, to see if better objective scores translate into better compliance of infection control measures by the illiterate workers in the long run.

\section{Conclusion}

Infection control programs using illiterate health care workers need new ideas for better implementation. This becomes extremely relevant in the ICUs of developing nations. Our study established significantly improved awareness of HAI amongst these workers after a visit to the microbiology laboratory with verbal and visual education. We also found some improvement in the practices of the illiterate workers thanks to enhanced awareness of HAI. Now we need to plan similar interventions to fill their awareness gaps in other areas.

\section{Acknowledgement:}

Dr SK Mandal, Head of Dept, Statistics, Centre for Biomedical Magnetic Resonance, SGPGIMS, Lucknow

\section{References}

1. Bhattacharya S, Mondal AS. Clinical microbiology in the intensive care unit: Strategic and operational characteristics. Indian J Med Microbiol 2010; 28: 5. http://dx.doi. org/10.4103/0255-0857.58720

2. Donskey CJ, Eckstein BC: Images in clinical medicine. The hands give it away. N Eng/ / Med 2009; 360: e3. http://dx.doi. org/10.1056/NEJMicm0707259

3. WHO and JHPIEGO document. Effective Teaching: A Guide for Educating Healthcare Providers. Module 1, Page 7. 2005. Available at www.jhpiego.org/resources/pubs/effteach/ EffTeach_man.pdf

4. Yassi A, Lockhart K, Copes R, et al. Determinants of Healthcare Workers' Compliance with Infection Control Procedures. Healthcare Quarterly 2007; 10(1): 44-52. 